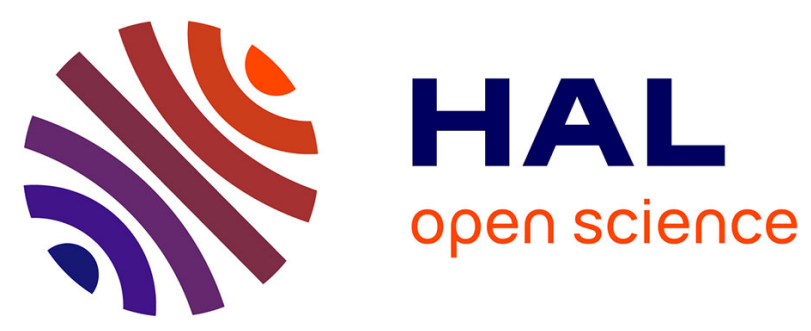

\title{
Some new trends on lithium niobate modulators
}

Nadège Courjal, M.-P. Bernal, J. Hauden, J. Amet, M. Roussey, H. Porte

\section{To cite this version:}

Nadège Courjal, M.-P. Bernal, J. Hauden, J. Amet, M. Roussey, et al.. Some new trends on lithium niobate modulators. Photonics West, Jan 2006, San-Jose, United States. pp.80-94, 10.1117/12.647820 . hal-00194328

\section{HAL Id: hal-00194328 \\ https://hal.science/hal-00194328}

Submitted on 9 Apr 2021

HAL is a multi-disciplinary open access archive for the deposit and dissemination of scientific research documents, whether they are published or not. The documents may come from teaching and research institutions in France or abroad, or from public or private research centers.
L'archive ouverte pluridisciplinaire HAL, est destinée au dépôt et à la diffusion de documents scientifiques de niveau recherche, publiés ou non, émanant des établissements d'enseignement et de recherche français ou étrangers, des laboratoires publics ou privés.

\section{(ㄷ)(1)}

Distributed under a Creative Commons Attribution| 4.0 International License 


\title{
Some new trends on lithium niobate modulators
}

\author{
Nadège Courjal ${ }^{* a}$, M.P. Bernal ${ }^{\mathrm{a}}$, J. Hauden ${ }^{\mathrm{b}}$, J. Amet ${ }^{\mathrm{a}}$, M. Roussey ${ }^{\mathrm{a}}$, H. Porte \\ Département Optique P.M. Duffieux, FEMTO-ST, UMR CNRS 6174, \\ ${ }^{\mathrm{a}}$ Université de Franche Comté, 25030 Besançon Cedex, France \\ ${ }^{\mathrm{b}}$ Photline Technologies, 25001 Besançon CEDEX 3, BP71025
}

\begin{abstract}
We report two novel kinds of $\mathrm{LiNbO}_{3}$ electro-optic modulators. The first one is oriented toward long haul high bit rate telecommunication systems. An original single-ended structure with a poled section and phase reversal electrodes is proposed to prevent the intensity modulation from chirp, without sacrifice on the driving voltage. We also show that improvements can be performed with the use of several poled sections. To remain attracting, $\mathrm{LiNbO}_{3}$ modulators should also exhibit a lower size. The second configuration described here is a new generation of $\mathrm{LiNbO}_{3}$ modulators based on photonic crystals, with a micrometric active length. We theoretically show that the optimal photonic structures for an efficient electro-optical tuning are based on a triangular array of holes integrated on a Xcut substrate. The first optical characterizations confirm the theoretical predictions, and exhibit a $-12 \mathrm{~dB}$ extinction ratio in the transmission response.
\end{abstract}

Keywords: Lithium niobate, electrooptic modulation, ferroelectric domain inversion, chirp, photonic crystal.

\section{INTRODUCTION}

Though based on a mature technology, $\mathrm{LiNbO}_{3}$ modulators are still the purpose of various researches, motivated by the numerous possibilities offered by the material. Examples can be quoted among new configurations for special modulation formats ${ }^{1}$, structures for wavelength conversion ${ }^{2}$, or for sensing applications ${ }^{3}$. $\mathrm{LiNbO}_{3}$ modulators are particularly attracting for telecommunication systems when the bit rates are high $(>10 \mathrm{~Gb} / \mathrm{s})$ and when the distances of transmission are long $(>50 \mathrm{~km})$. Indeed their low optical losses, large extinction ratio and small wavelength dependence make $\mathrm{LiNbO}_{3}$ modulators extremely suitable for Dense Wavelength Multiplexing (DWDM) and Optical Time Division Multiplexing (OTDM) long haul transmission systems. $\mathrm{LiNbO}_{3}$ modulators exhibit the additional advantage of large bandwidth, zero or tunable chirp and low driving voltage. But the gathering of all these advantages in the same device remains a challenging task ${ }^{4-9}$.

Basically, two kinds of $\mathrm{LiNbO}_{3}$ optical modulators can be distinguished. On the one hand, $\mathrm{X}$-cut devices benefit from a zero chirp parameter, which is very important in long haul transmission systems. Indeed, a low chirp parameter can limit the distortions of the signals propagating through dispersive fibers. But single-ended X-cut modulators exhibit a typical DC driving voltage of $6 \mathrm{~V}$. The use of thin layers of X-cut substrate ${ }^{5,6}$ can yield a lower driving voltage, but this implies non trivial additional steps in the fabrication process. On the other hand, Z-cut modulators can allow a low driving voltage (typically 4V), but they are affected by frequency chirping, due to the asymmetry of their structure. Push-pull drive $\mathrm{p}^{7}$ modulators represent an interesting alternative to allow both a control of the chirp and a low driving voltage. However, the $40 \mathrm{~Gb} / \mathrm{s}$ input signals have to be differentially adjusted, which is complex when the bit rate is larger than $10 \mathrm{~Gb} / \mathrm{s}$.

Here we show that an original structure based on ferroelectric domain inversion associated with phase reversal electrodes can allow both a chirp-free modulation and a low driving voltage with one single electrode. Then we describe improvements that can be derived from this structure.

To become more attracting, $\mathrm{LiNbO}_{3}$ modulators should also feature a smaller size. The typical size of $\mathrm{LiNbO}_{3}$ electro-optical modulators is $6 \mathrm{~cm}$, which is due to a very long active length. Here we report an original solution, based on photonic crystal structures. The aim is to show the feasibility of $\mathrm{LiNbO}_{3}$ photonic crystal, by presenting the first theoretical and experimental results.

To understand the originality of the devices presented here, we firstly describe briefly the main principles of standard electro-optic $\mathrm{LiNbO}_{3}$ modulators. 


\section{CONVENTIONAL SINGLE ENDED ELECTRO-OPTICAL MODULATORS}

$\mathrm{LiNbO}_{3}$ Mach-Zehnder modulators are based on the electro-optical effect, which consists in inducing a change in the refractive index by means of an external electric field. As the $r_{33}$ coefficient is the largest electro-optical coefficient of $\mathrm{LiNbO}_{3}$ materials $\left(r_{33}=30.8 \mathrm{pm} / \mathrm{V} @ 1550 \mathrm{~nm}\right)$, it is desirable to apply the electrical field along the Z-direction of the crystal. If the light is also polarized along the Z-axis, then the change in the extraordinary refractive index $n_{e}$ can be expressed as:

$$
\Delta n_{e}=\frac{-1}{2} n_{e}^{3} \cdot r_{33} \cdot\langle F\rangle \text { (1), }
$$

which is the largest change that can be performed with a $\langle F\rangle$ effective electric field in a $\mathrm{LiNbO}_{3}$ substrate. The effective electric field can also be evaluated in term of the applied voltage $V$ as $\langle F\rangle=\eta V / g$ where $g$ is the space between the two transmission lines, and $\eta$ is the overlap coefficient between the electric field and optical field.

Basically, two kinds of configurations rely on the electro-optical effect. The first configuration corresponds to modulators integrated on X-cut substrates. In this case, the Z-axis is parallel to the surface of the wafer. Therefore, an electric field along the Z-direction implies electrodes that are deposited side by side with the optical waveguides. The electrode configuration is illustrated in figure 1(a). The optical waveguide has to be along the Y-axis, and a TE polarization is recommended in order to benefit from the $r_{33}$ coefficient. In the second conventional configuration, the modulator is integrated on a Z-cut substrate: the Z-axis is normal to the surface of the wafer and the light has to be TM-polarized. One of the optical branches of the Mach-Zehnder modulator is placed under the central line, while the other branch is located under one of the two lateral ground lines, as schematically depicted in figure 1(b).

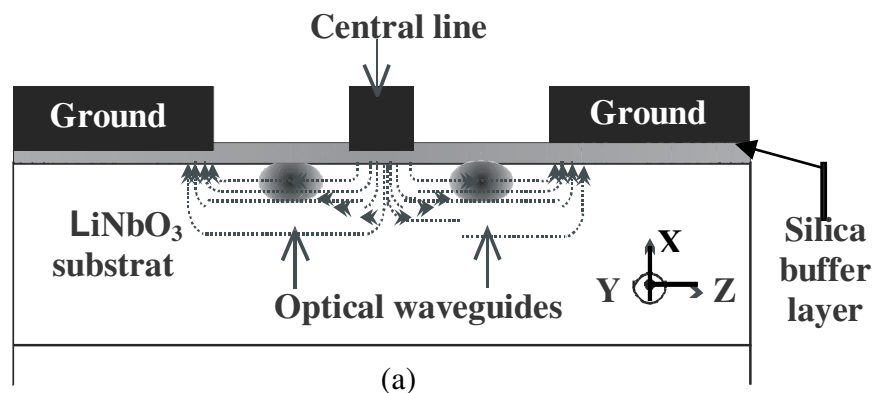

Figure 1: Transversal structure of : (a) $\mathrm{X}$-cut $\mathrm{LiNbO}_{3}$ modulators, (b) Z-cut $\mathrm{LiNbO}_{3}$ modulators

The two following sections are devoted to two new kinds of structures based on the electro-optical effect, for reducing either the chirp or the size of the conventional single ended structures.

\section{LOW CHIRP MACH ZEHNDER MODULATORS WITH ONE OR SEVERAL FERROELECTRIC DOMAIN SECTION}

The first configuration described here is based on coplanar waveguide electrodes designed to meet the requirements of long haul transmission systems. We propose a simple structure that enables both a control of the chirp parameter and a low driving voltage, by means of a poled section associated with phase reversal electrodes. The basic principles of the structure are given in ref. ${ }^{10}$ and ${ }^{11}$. Here we remind these principles, shows new results and propose new improvements.

\subsection{Principle of the device}

The chirp parameter of a modulator denotes its ability to modulate the phase simultaneously with the intensity of the output light. A non-zero chirp parameter appears when the two branches of the Mach-Zehnder interferometer are not submitted to the same effective electric field. In ref. ${ }^{11}$, we have shown that it can be expressed at small signal regime and quadratic bias as (2): 


$$
\alpha_{\pi / 2}=\frac{\dot{\varphi}_{1}(t)+\dot{\varphi}_{2}(t)}{\dot{\varphi}_{1}(t)-\dot{\varphi}_{2}(t)}
$$

where $\varphi_{1}$ and $\varphi_{2}$ denote the cumulated phase at the outputs of the Mach-Zehnder structure, and the superscript ${ }^{\bullet}$ represents time derivative. In the push-pull configuration, $\dot{\varphi}_{1}$ and $\dot{\varphi}_{2}$ are of opposite signs. They are proportional to $\eta_{1}$ and $\eta_{2}$, which are the overlap coefficient between electric and optical field in branch \#1 and \#2 respectively. Contrary to Z-cut modulators, X-cut modulators (figure 1(b)) exhibit a symmetric configuration, $\dot{\varphi}_{1}=-\dot{\varphi}_{2}$ which enables a chirp free modulation. But the push-pull configuration of a Z-cut modulator can yield a lower driving voltage than that of $\mathrm{X}$-cut modulators. To provide both a chirp free modulation and a low driving voltage, two ways can be explored. The first possible way consists in reducing the driving voltage of an X-cut modulator. Recent studies have shown the feasibility of this approach by means of thin layers that enhance the effective electric field seen by the optical waveguides ${ }^{5,6 .}$ This is however difficult to fabricate with batch methods.

The second approach consists in reducing the chirp parameter of a Z-cut modulator. The origin of chirp in Z-cut single-ended modulators is linked to the asymmetry between the two optical branches: the branch placed under the central line is submitted to a $\eta_{C}$ overlap coefficient that is higher in amplitude that the $\eta_{G}$ overlap coefficient relative to the optical branch located under one of the lateral ground lines. This asymmetry causes the chirp value at quadratic bias to be:

$$
\alpha_{\pi / 2}^{S}=\frac{\eta_{G}-\eta_{C}}{\eta_{G}+\eta_{C}}
$$

Ferroelectric domain inversion appears as an attractive means for ensuring symmetry between the two optical arms of the interferometer. In ref ${ }^{9}$, Oikawa and al. use a reversal branch with one central line divided into two parts. This configuration features a low chirp parameter for a wide range of frequencies, but this is obtained at the expense of impedance matching. In ref. ${ }^{10,11}$ we have proposed an alternative method based on two sections of opposite domains and reversal electrodes. The principle is schematically depicted in figure 2: a poled section is associated with a phase reversal electrode section in order to make the structure symmetric without sacrifice on the modulation efficiency. If the length $L_{1}$ of the first section is equal to the length $L_{2}$ of the second section, symmetry is established between the two arms of the Mach-Zehnder modulator (MZM). The chirp parameter is thus set to zero if the electrical losses are assumed to be negligible.

However, a low driving voltage requires a long active length. In this case, an RF attenuation of the electrical field in the line can induce a significant asymmetry between the two arms of the Mach-Zehnder, and produce a residual chirp at high frequencies. Here we propose to model and experimentally verify the frequency dependence of chirp. Finally, we will propose solutions to avoid or control the frequency chirping.

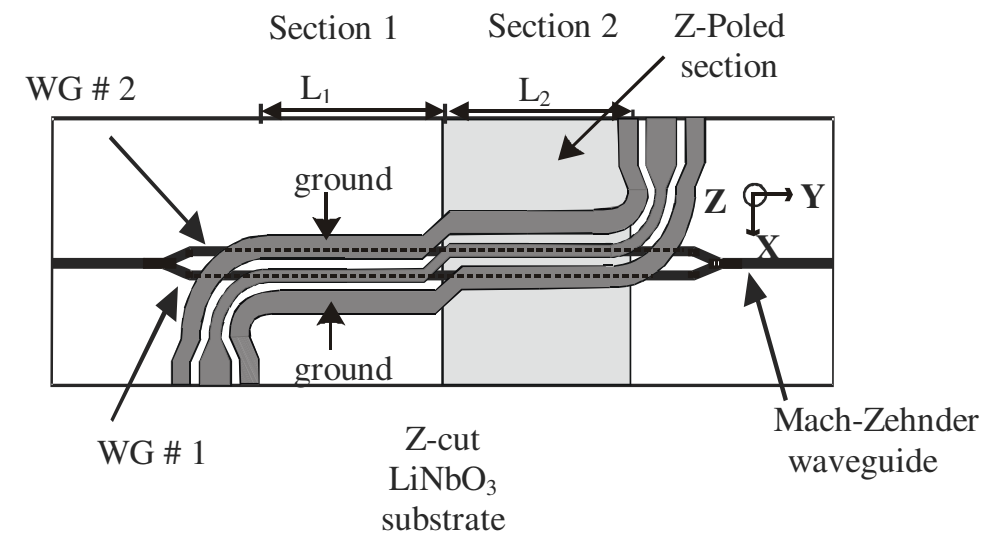

Figure 2: Structure of a Z-cut MZ modulator with inverted domain and phase reversal electrode

A voltage $V z$ is applied to the electrodes. Due to the association of ferroelectric domain inversion with the phase reversal inversion of electrodes, the optical phase of a light beam of wavelength $\lambda$ accrued through each of the branches of the Mach-Zehnder modulator (MZM) can be expressed as, respectively: 


$$
\begin{aligned}
& \varphi_{1}=K \cdot V_{Z} \cdot\left(\gamma_{1} \cdot \eta_{C} \cdot L_{1}-\gamma_{2} \cdot \eta_{G} \cdot L_{2}\right), \\
& \varphi_{2}=K \cdot V_{Z} \cdot\left(\gamma_{1} \cdot \eta_{G} \cdot L_{1}-\gamma_{2} \cdot \eta_{C} \cdot L_{2}\right),
\end{aligned}
$$

where $\gamma_{1}$ and $\gamma_{2}$ account for the lossy transmission of the electrical signal through the optical path in region 1 and 2 respectively (see eqs (6) and (7) for more details). $K=\pi n_{e}^{3}\left|r_{33}\right| / \lambda g$ is a constant related to the extraordinary refractive index $n_{e}$ of lithium niobate and to the electrode gap $g$. For simplicity, (4) and (5) have been derived assuming the electrical index is matched to the optical refractive index, which is a condition required in high-speed modulators.

The chirp parameter at small signal regime and quadratic bias is deduced from equation (1), (2), (3), (4) and (5) :

$$
\alpha_{\pi / 2}=\frac{\left(\gamma_{1} L_{1}-\gamma_{2} L_{2}\right)}{\left(\gamma_{1} L_{1}+\gamma_{2} L_{2}\right)} \times \frac{\left(\eta_{C}+\eta_{G}\right)}{\left(\eta_{C}-\eta_{G}\right)}=\frac{\left(\gamma_{1} L_{1}-\gamma_{2} L_{2}\right)}{\left(\gamma_{1} L_{1}+\gamma_{2} L_{2}\right)} \times \alpha_{\pi / 2}^{S}
$$

The electrical losses in section 1 and 2 can be modelled by an attenuation coefficient $a_{0}$, that stands for the electrical losses in $\mathrm{Np} / \mathrm{GHz}^{0.5} / \mathrm{cm}$ :

$$
\begin{aligned}
& \gamma_{1}=\frac{1}{L_{1}} \int_{0}^{L_{1}} \exp \left(-a_{0} f^{1 / 2} z\right) d z \\
& \gamma_{2}=\frac{1}{L_{2}} \int_{L_{1}}^{L} \exp \left(-a_{0} f^{1 / 2} z\right) d z
\end{aligned}
$$

The intrinsic chirp parameter is represented in figure 3 for $L_{1}=L_{2}=1.4 \mathrm{~cm}$ and different cases of electrical losses. According to these results, a propagation loss of $0.057 \mathrm{~Np} / \mathrm{GHz}^{0.5} / \mathrm{cm}\left(\right.$ e.g. $\left.0.48 \mathrm{~dB} / \mathrm{GHz}^{0.5} / \mathrm{cm}\right)$ yields a variation of the chirp parameter that is lower than 0.1 at $10 \mathrm{GHz}$.

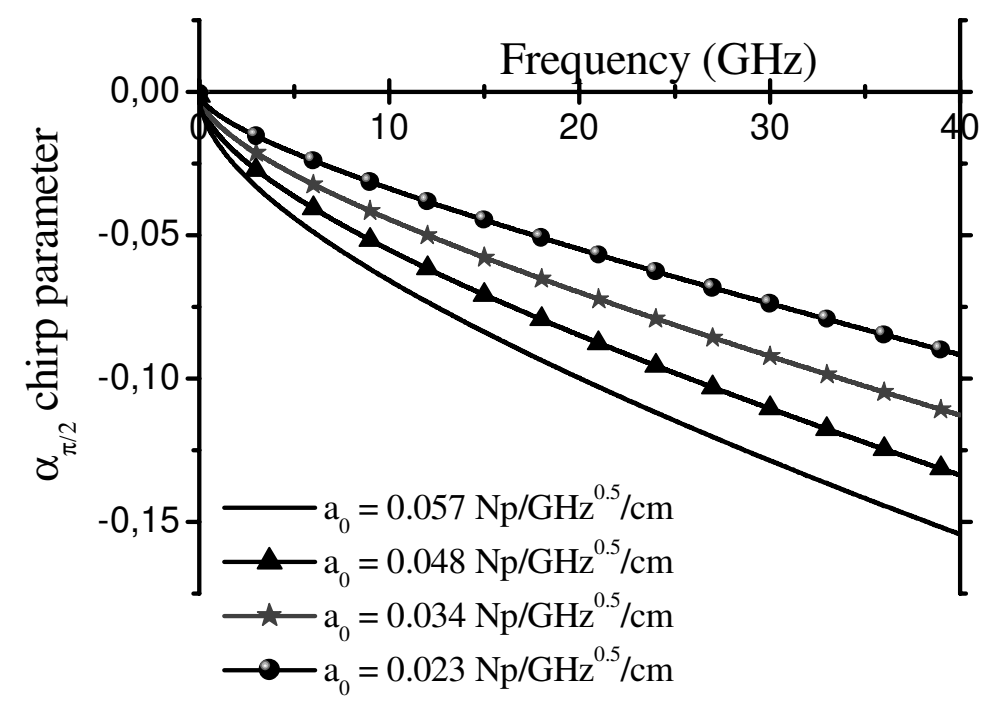

Figure 3: Variation of the intrinsic chirp parameter $\alpha_{\pi / 2}$ with frequency for different cases of electrical attenuation

With a proper $\mathrm{L}_{1} / \mathrm{L}_{2}$ ratio the chirp parameter can even be forced to zero for a given frequency if the second section sufficiently long to compensate for electrical losses. It is also worth noting that the theoretical difference of phase $\Delta \varphi=\varphi_{1}-\varphi_{2}$ induced between the two optical paths is exactly the same as that induced in a conventional single ended modulator. So the structure should exhibit the same driving voltage as that of conventional Z-cut single ended modulators, with a significantly lower chirp parameter.

\subsection{Experimental results}

A modulator with an inverted ferro-electric domain section was fabricated to provide a low driving voltage, low electrical losses and index matching. The $L_{1} / L_{2}$ ratio was chosen to be equal to 0.87 in order to set the chirp 
parameter to zero at $5 \mathrm{GHz}$ (cf eq.(6) and (7)), which corresponds to the frequency carrier of a NRZ modulation at $10 \mathrm{Gbit} / \mathrm{s}$ bit rate. The different steps of realization are described in ref ${ }^{10}$.

Measured total fiber to fiber insertion loss was $-3.9 \mathrm{~dB}$. The static half wave voltage at $1550 \mathrm{~nm}$ is measured to be $4.5 \mathrm{~V}$, and the extinction ratio is $-25 \mathrm{~dB}$. The electrical attenuation was determined from the $\mathrm{s}_{21}$ electrical response: $a_{d B}=0.48 \mathrm{~dB} / \mathrm{GHz}^{0.5} / \mathrm{cm}$. The $3 \mathrm{~dB}$ e-o bandwidth was $14 \mathrm{GHz}$ measured using a vectorial network analyser and a calibrated detector (Fig. 4).
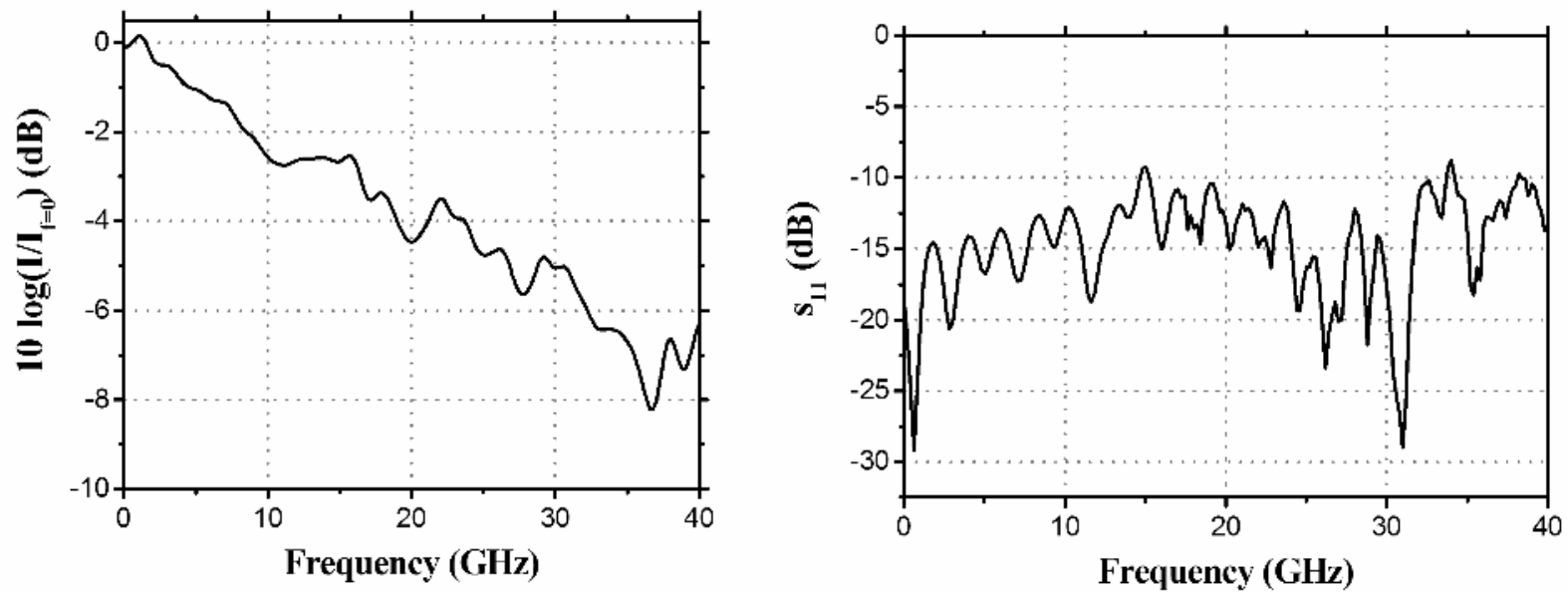

Figure 4: electro-optical transmission response

$10 \mathrm{~Gb} / \mathrm{s}$ and $40 \mathrm{~Gb} / \mathrm{s} \mathrm{NRZ}$ transmission through the poled Z-cut modulator were carried out with $2^{31}-1$ frame pattern. The results are exposed in figure 5 . At $10 \mathrm{~Gb} / \mathrm{s}$ bit rate, the optical signal exhibits a clear open eye (>85\%) (figure $5(\mathrm{a})$ ). The $10 \mathrm{~Gb} / \mathrm{s}$ measurements were performed with a detector with $12 \mathrm{GHz}$ bandwidth and a driver with $20 \mathrm{GHz}$ bandwidth and $22 \mathrm{~dB}$ gain. The $40 \mathrm{~Gb} / \mathrm{s}$ eye diagram was evaluated with a $40 \mathrm{GHz}$ detector and a driver with $20 \mathrm{~dB}$ gain and $40 \mathrm{GHz}$ bandwidth. The results in figure $5(\mathrm{~b})$ show that the eye diagram remains largely open $(>50 \%)$ at $40 \mathrm{~Gb} / \mathrm{s}$ bit rate.

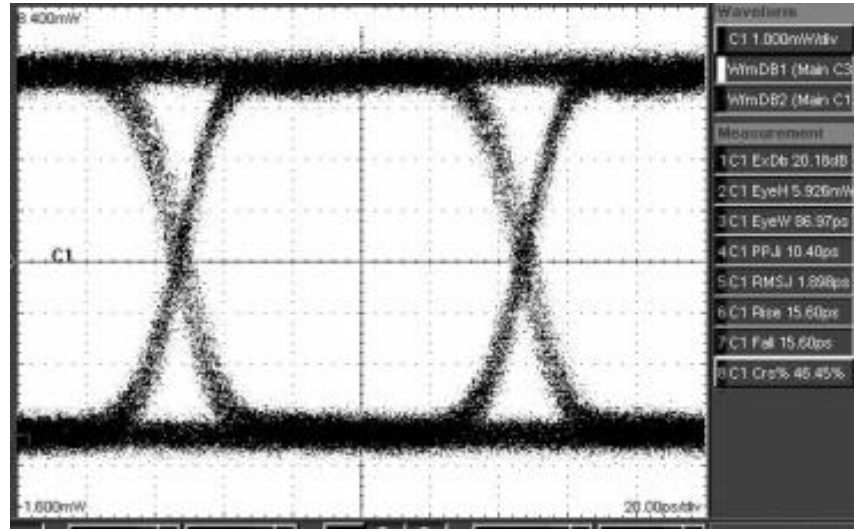

(a)

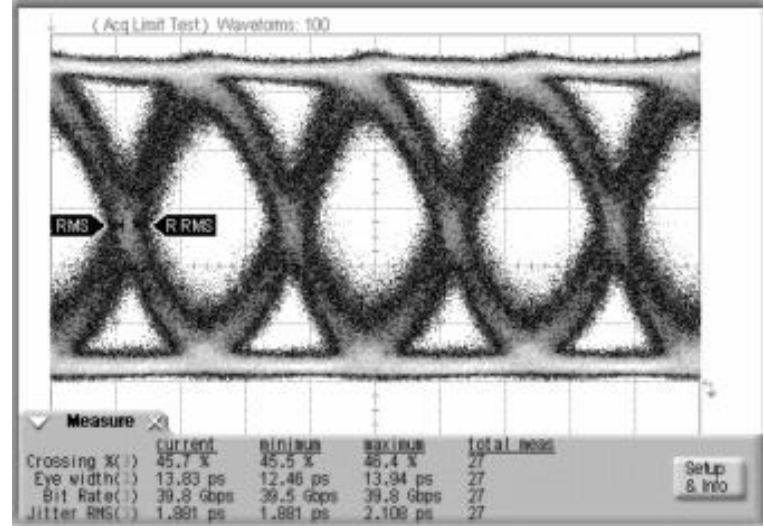

(b)

Figure 5: back-to-back eye diagram at the output of the poled Z-cut modulator. (a) $10 \mathrm{~Gb} / \mathrm{s}$, (b) $40 \mathrm{~Gb} / \mathrm{s}$. 


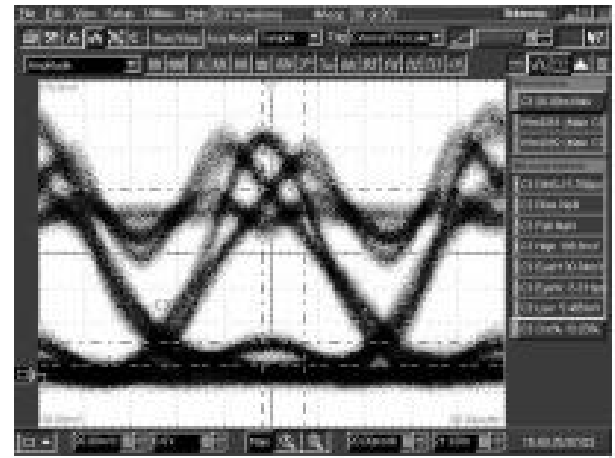

(a) Z-cut poled, $\mathrm{V}_{\pi}>0$

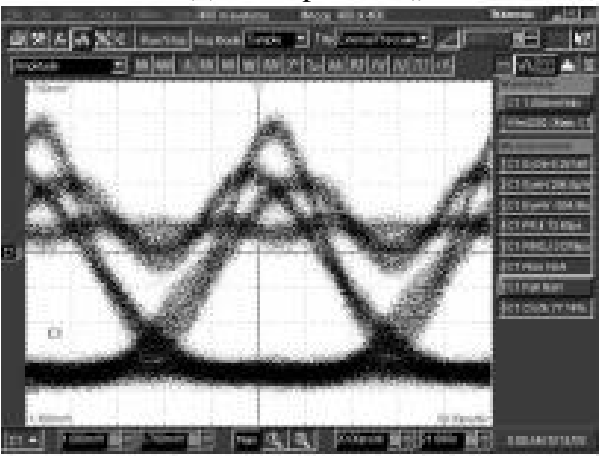

(c) X-cut, $\mathrm{V}_{\pi}>0$

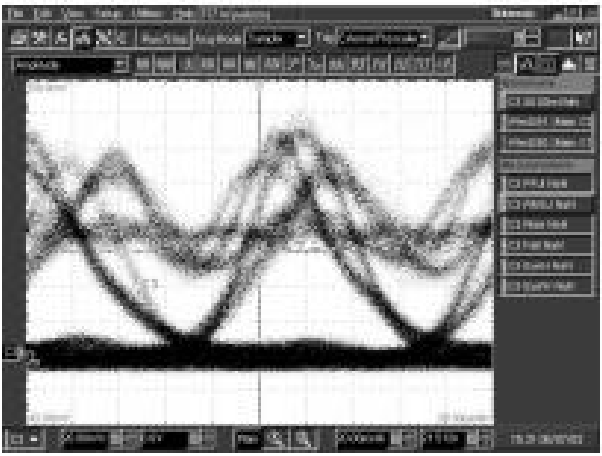

(e) Z-cut, $V_{\pi}>0$

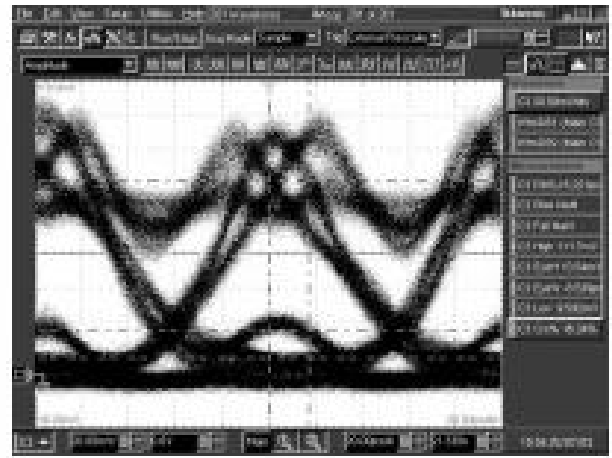

(b) Z-cut poled, $\mathrm{V}_{\pi}<0$

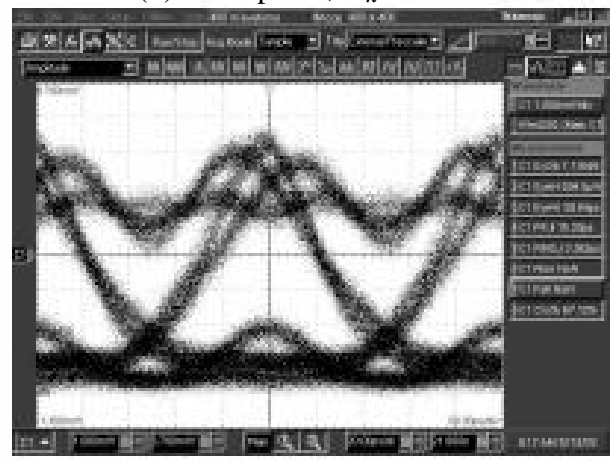

(d) X-cut, $\mathrm{V}_{\pi}<0$

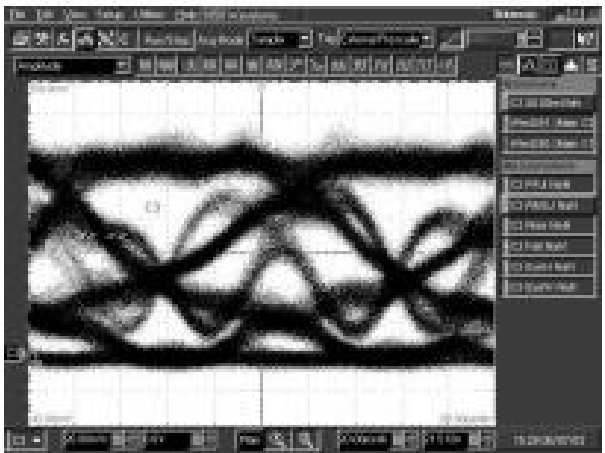

(f) Z-cut, $\mathrm{V}_{\pi}<0$

Figure 6: eye diagrams after $50 \mathrm{~km}$ of propagation through a dispersive fiber (SMF28)

$10 \mathrm{~Gb} / \mathrm{s}$ NRZ transmission was then performed through 50kms of standard fiber (SMF 28, D=17ps/nm/km). Figure 6 shows the resulting eye diagrams for a positive and a negative DC bias for three kinds of modulators : the first modulator is the Z-poled modulator (fig. 6(a) and fig. 6(b)), the second one is an X-cut modulator (fig. 6(c) and fig. $6(\mathrm{~d})$ ), and the third one is a Z-cut modulator realized in the same condition than the first one, with the same active length, but with only one section (standard Z-cut single ended modulator) (fig. 6(e) and fig. 6(f)). To interpret these results, we should first outline that the sign of the chirp parameter is dependant on the sign of the bias. This property can be exploited to emphasize the frequency chirping of the three modulators under test. Indeed, if a modulator exhibits a low chirp parameter, then the propagation through $50 \mathrm{kms}$ of dispersive fiber is not modified by the change of the sign of bias. Conversely, a non-zero chirp parameter will be detected by a modification of the eye diagram after $50 \mathrm{kms}$ of propagation, due to the change of the bias' sign. It can be deduced from figure 6 that the X-cut modulator and the Z-poled modulator exhibit a very low frequency chirping, contrary to the "conventional" Z-cut modulator.

So the Z-poled modulator can be compared to a X-cut modulator in term of chirp, and to a Z-cut modulator in term 
of driving voltage $(4,5 \mathrm{~V})$.

The third step of experimental verifications consisted in measuring the frequency chirping of the presented modulator, in order to complete the results of ref ${ }^{11}$. The measurements were performed by optical frequency discrimination, as described in ref. ${ }^{12}$.

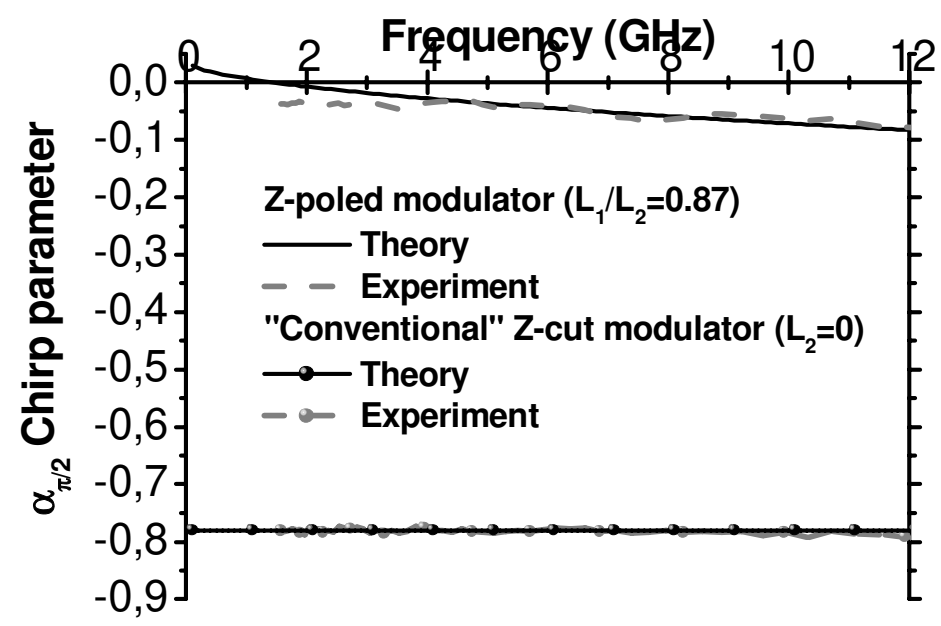

Figure 7: experimental and theoretical frequency chirping of the Z-poled modulator, comparison with a standard Z-cut single ended modulator

It is clear from figure 7 that the frequency chirping of the Z-poled modulator (solid black line) is far lower than that of a conventional Z-cut single ended modulator (solid black line with circle). However, the frequency dependence of chirp is confirmed, which could be harmful for $40 \mathrm{~Gb} / \mathrm{s}$ and higher rates. A simple solution based on several reversal sections is proposed in what follows to fight against this disadvantage.

\subsection{Z-poled modulator with two reversal sections}

A modulator with two ferroelectric domain sections is depicted in figure 8. The structure is quite similar to that of figure 2, except that two other sections are added beyond the second section. The third section is similar to the first one. It is a non inverted section, with a length equal to $L_{3}$. The length of the fourth zone is $L_{4}$. This section is a ferroelectric domain inverted section, similar to the second section.

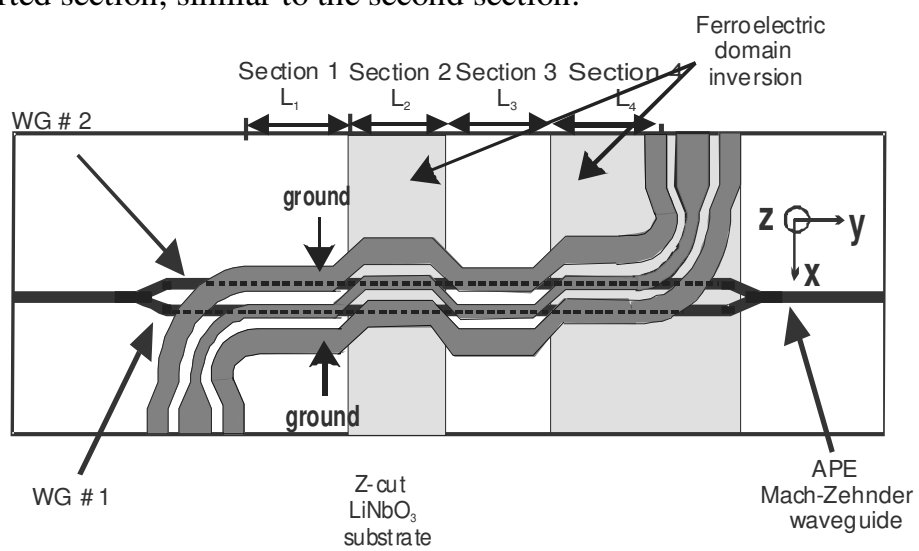

Figure 8: Z-cut modulator with two reversal sections

The $\alpha_{\pi / 2}$ intrinsic chirp parameter of this new configuration can be evaluated by equation (9), which is very similar to equation (6):

$$
\alpha_{\pi / 2}=\frac{\left(\gamma_{1} L_{1}-\gamma_{2} L_{2}+\gamma_{3} L_{3}-\gamma_{4} L_{4}\right)}{\left(\gamma_{1} L_{1}+\gamma_{2} L_{2}+\gamma_{3} L_{3}+\gamma_{4} L_{4}\right)} \times \alpha_{\pi / 2}^{S}
$$


where $\gamma_{i}$ accounts for the contribution of electrical losses through the optical path, as previously described. $\gamma_{i}$ can be derived as :

$$
\gamma_{i}=\exp \left(-a_{0} \sqrt{f} \sum_{p=1}^{i-1} L_{p}\right) \cdot \frac{\exp \left(-a_{0} \sqrt{f} L_{i}\right)-1}{-a_{0} \sqrt{f}}
$$

The purpose of this theoretical approach is to minimize the frequency dependence of chirp by choosing appropriate lengths $L_{1}, L_{2}$ and $L_{3}$. The length $L_{4}$, is then determined from: $L_{4}=L-\left(L_{1}+L_{2}+L_{3}\right)$. The first condition is achieved by setting the $\alpha_{\pi / 2}$ parameter to zero at low frequencies. This condition implies that the total length of the poled sections is identical to the total length of the non inverted sections:

$$
L_{1}+L_{3}=L_{2}+L_{4}=L / 2
$$

The second condition is obtained by minimizing $\mathrm{d} \alpha_{\pi / 2} / \mathrm{d} f$ at low frequencies and leads to:

$$
L_{3} \cdot L_{2}=L^{2} / 8
$$

So conditions (11) and (12) impose a minimal variation of $\alpha_{\pi / 2}$ around zero at low frequencies.

The final aim of this study consists in minimizing the frequency dependence of $\alpha_{\pi / 2}$ for a wide frequency range. This is done by choosing $L_{1}$ so that $\alpha_{\pi / 2}=0$ for a proper frequency:

$$
L_{1}=\frac{-1}{a_{0} \sqrt{f}} \cdot \frac{1+\exp \left(-a_{0} \sqrt{f} L\right)-2 \cdot \exp \left(-a_{0} \sqrt{f}\left(L-L_{4}\right)\right)}{2 \cdot\left(1-\exp \left(-a_{0} \sqrt{f} L\right)\right)}
$$

This equation emphasizes the interest of using four adjacent sections instead of two: a modulator with 4 sections can offer the possibility of controlling the frequency chirping in a stronger way than that provided by a two sections configuration.

The systems of equations (11), (12) and (13) can be solved by the least mean square method, taking $a_{0}=0.057$ $\mathrm{Np} / \mathrm{GHz}^{1 / 2} / \mathrm{cm}$ electrical losses and a total length $L=2.8 \mathrm{~cm}$. The resulting lengths are calculated to be: $L_{l}=3.92 \mathrm{~mm}$, $L_{2}=9.73 \mathrm{~mm}, L_{3}=10.08 \mathrm{~mm}$ and $L_{4}=4.27 \mathrm{~mm}$. The dotted lines in figure $4(\mathrm{a})$ and (b) represent the variation of $\alpha_{\pi / 2}$ in these conditions. $\alpha_{\pi / 2}$ is equal to zero at $30 \mathrm{GHz}$ and $0 \mathrm{~Hz}$, and the chirp parameter remains in the $\left[-2 \times 10^{-4}, 2 \times 10^{-4}\right]$ interval for a frequency range of $40 \mathrm{GHz}$, which is more than one thousand times lower than the chirp of a standard Z-cut modulator. Moreover, the addition of a new reversal section should play in favor of stability. In fact, a poled section reduces the built up of charge induced by the pyroelectric effect.

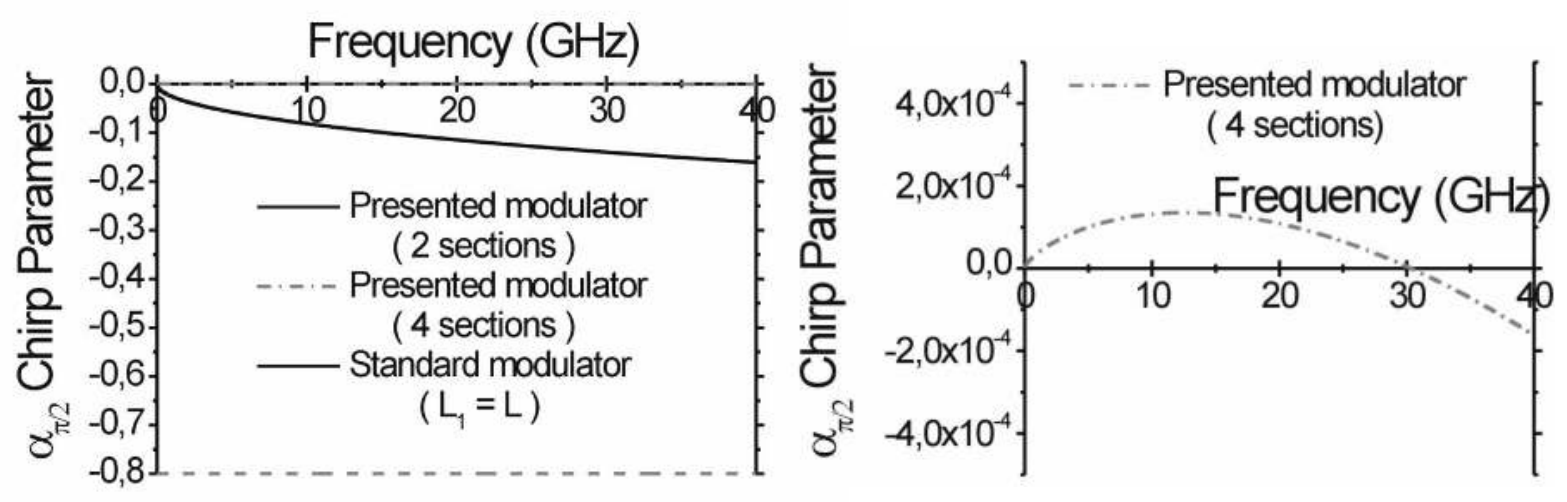

Figure 9: Theoretical evaluation of the frequency chirping of a conventional Z-cut single-ended modulator (dashed gray line), and comparison with a Z-poled modulator with one section (solid black line) and four sections (dotted dashed gray line)

So we have shown that the dispersion of the intrinsic chirp parameter can be controlled by an appropriate choice of the lengths of the sections, which can be very useful to inhibit the distortion of signals propagating at high transmission rates.

The above configuration is once again a demonstration of the maturity of $\mathrm{LiNbO}_{3}$ technology and knowledge, which is much appreciated in telecommunication systems. However, a big disadvantage can be outlined in disfavour of 
$\mathrm{LiNbO}_{3}$ modulators. Indeed, their typical size is largely over the centimetre, which is very big in comparison with the millimetre size of electro-absorbent modulators. In the following section, we show that a new approach based on photonic crystal allows a considerable reduction of the size of conventional modulators.

\section{LiNbO $_{3}$ PHOTONIC CRYSTAL: TOWARD COMPACT ELECTRO-OPTIC MODULATORS}

Photonic crystals appear as an appealing feature of confining the light in small volumes. This confinement can be exploited to reduce the active length of electro-optic modulators. In this paper, we show that a photonic crystal integrated on a $\mathrm{LiNbO}_{3}$ substrate enables a significant reduction of $\mathrm{LiNbO}_{3}$ modulators' size. We report here the theory and the principal first results. The results relative to the electro-optical tuning will be presented at the oral conference.

\section{1 $\mathrm{LiNbO}_{3}$ photonic crystals}

Photonic crystals (PC) can be described as a periodic change of the dielectric constant on a length scale comparable to optical wavelengths. Their behaviour can be directly compared to the one of electrons in an atomic crystalline potential. Multiple interferences between scattered light waves can eventually lead to some frequencies that are not allowed to propagate, giving rise to forbidden and permitted bands, analogous to the electronic band gap of a semiconductor. Many experimental efforts have been devoted to PC devices due to the possibility of producing optical circuits with a micrometric size. Among the attracting applications, the fabrication of $\mathrm{LiNbO}_{3}$ photonic crystals offers the interest of reducing the size of standard modulators, and opens the way towards a wide range of compact dynamic components, like dynamic filters or couplers. These prospects have stimulated the research on nano-structuring lithium niobate materials ${ }^{13-15}$. However, the realization of good nanometric optical structures continues to be a difficult task due to its well known resistance towards standard machining techniques like wetetching. Till now, focused ion beam has shown to be the most indicated technique to produce nanometric size holes with high aspect ratio ${ }^{13,14}$. Here we describe how this performance can lead to the fabrication of compact $\mathrm{LiNbO}_{3}$ modulators. Firstly, we are going to show which configurations are potentially candidates for the fabrication of compact $\mathrm{LiNbO}_{3}$ modulators. Then we will describe fabrication and finally, we will show our first experimental results.

\subsection{Theory}

The proposed compact $\mathrm{LiNbO}_{3}$ modulators are based on photonic crystal structures that feature photonic band gaps. The modulation of the refractive index is expected to induce a modulation of the gap, which causes modulation intensity at the output of the periodic structure. So a periodic array that features gaps in its band structure can become a modulator if it is submitted to an external electric field that modulates the refractive index. As described in section 1, the electro-optic effect is optimal in $\mathrm{LiNbO}_{3}$ substrates if the electric field and the polarization of light are applied along the $\mathrm{Z}$ axis of the substrate. So we will only consider two kinds of configurations: a TE-polarized light propagating in a X-cut substrate (fig.10(a)) and a TM-polarized light propagating in a Z-cut substrate (fig. 10(b)). The light is assumed to be vertically confined with an optical waveguide performed by annealed proton exchange (APE). The light is launched into a 2D periodic arrangement of holes or rods, as depicted in figure 10. The position of the electrodes over the waveguides is the same as shown in figure 1. Indeed, the optical waveguide and its photonic structure are placed between the electrodes in case of a X-cut modulator (fig. 10(a)), while they are located under the central electrode in case of a Z-cut modulator (fig. 10(b)).

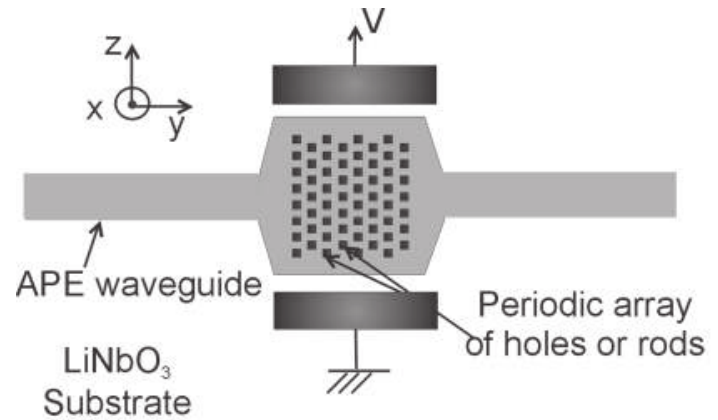

(a)

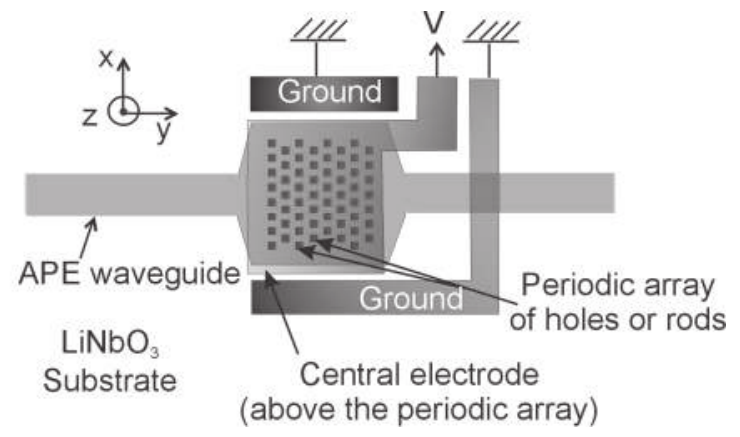

(b)

Figure 10: Schematic view of photonic crystal modulators integrated on a X-cut $\mathrm{LiNbO}_{3}$ substrate (a), or on a Z-cut $\mathrm{LiNbO}_{3}$ substrate (.b) 
With adequate properties of the periodic arrangement, a photonic band gap (PBG) can be expected. The objective is firstly to determine which periodic patterns can allow a photonic band gap. The photonic band structures are numerically evaluated with the bandsolve software that lies on the plane wave expansion method. According to the results, only two patterns can exhibit a photonic band gap: the first one is a triangular arrangement of holes in a Z-cut substrate, and the second one is a square arrangement of rods in a X-cut substrate (fig. 11(a)). We will only expose here the results relative to the X-cut substrate, because it would be difficult to deposit electrodes above rods integrated on the Z-cut substrate. The theoretical band diagram of the triangular array of holes is represented in figure 11(b), and the pattern is schematically depicted in figures 11(a). The minimal diameter of holes that allows a photonic bandgap in a triangular arrangement of holes was calculated to be $0.34 a$, where $a$ is the periodicity. The choice of a $d / a=0.5$ ratio in our calculations is motivated by technological reasons.

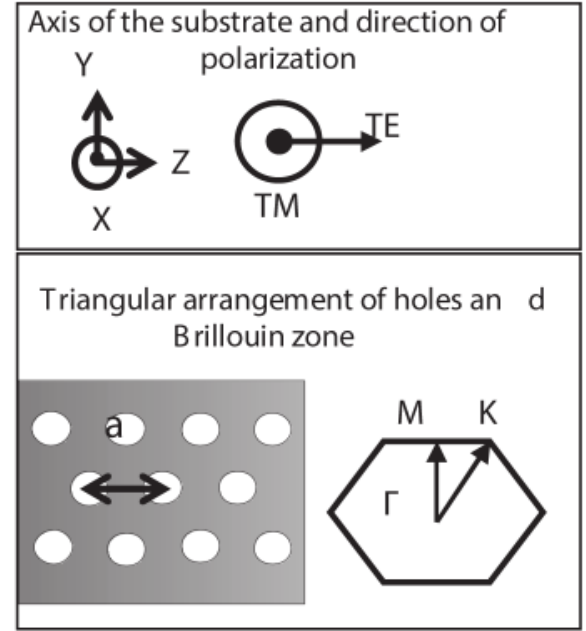

(a)

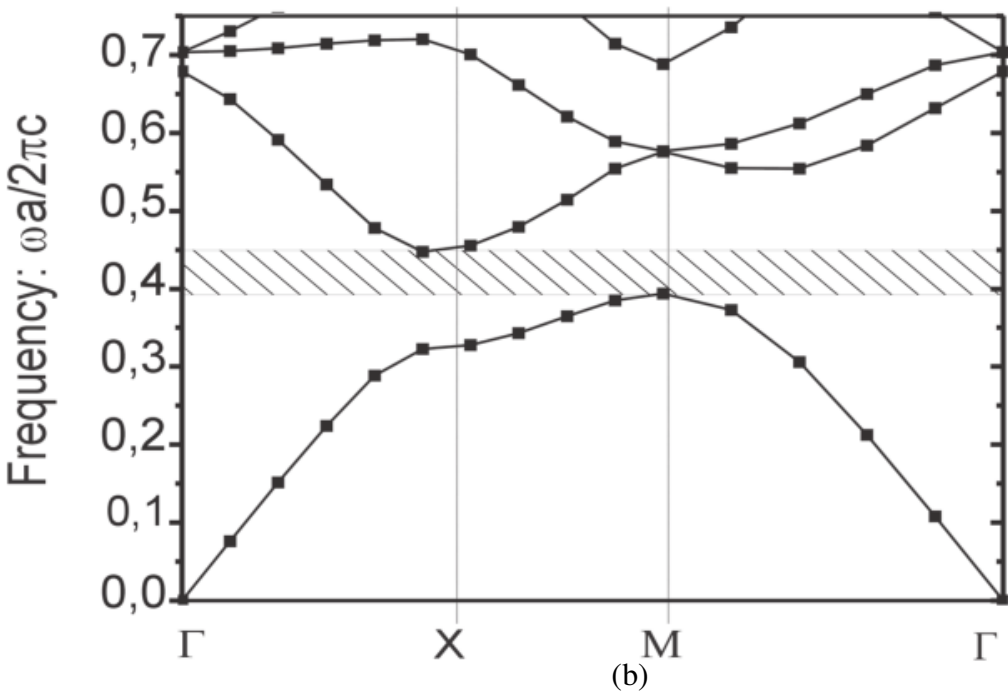

(b)

Figure 11: (a) Schematic view of a triangular arrangement of holes, and its Brillouin zone. (b) Band diagram of atriangular arrangement of holes in an $\mathrm{X}$-cut $\mathrm{LiNbO}_{3}$ substrate . $(\mathrm{d} / \mathrm{a}=0,5)$.

The spectral position of the gap is dependant on the refractive index. So the transmission response can be modulated if the working wavelength corresponds to an edge of the band gap. The aim is to determine which direction of propagation is the most appropriate to move the optical gap by modulating the refractive index. To evaluate the optimal direction of propagation, we have numerically calculated the transmission response by means of bidimensional finite difference time domain method (FDTD2D). The results are exposed in table I for a triangular array of holes in an X-cut substrate.

\begin{tabular}{cccccc}
\hline $\begin{array}{c}\text { Propagation } \\
\text { direction }\end{array}$ & $\begin{array}{c}\text { FWHM } \\
(\mathrm{nm})\end{array}$ & $\begin{array}{c}\text { Tmin } \\
(\%)\end{array}$ & $\begin{array}{c}\mathrm{a} / \lambda \min \\
(\mathrm{nm})\end{array}$ & $\begin{array}{c}\mathrm{a} / \lambda_{\text {FWHM }} \\
(\mathrm{nm})\end{array}$ & $\eta=\Delta \lambda / \Delta \mathrm{n} / \mathrm{a}$ \\
\hline$\Gamma \mathrm{K}$ & 233 & $3,4.10^{-2}$ & 0,3518 & 0,3259 & 1,41 \\
$\Gamma \mathrm{M}$ & 316 & $3,7.10^{-7}$ & 0,3105 & 0,2788 & 1,63 \\
\hline
\end{tabular}

Table I: Numerical study of the optical transmission of a TE polarized light through a photonic structure of 21 periods of holes in a triangular lattice. X-cut substrate, Y-propagation, Z-polarization (TE)

We have called $T_{\min }$ the smallest value of the transmission spectrum and $\lambda_{\min }$ its corresponding wavelength. $\lambda_{F W H M}$ is the wavelength at the right band gap edge for the Full Width Half Maximum (FWHM) transmission value. We denote $\eta$ the slope of the linear function $\Delta \lambda / a=\mathrm{f}(\Delta n)$. This coefficient gives information on the sensitivity of the PBG for a change in the index of refraction. It is clear from the tables' results that the optimal sensitivity is obtained when the propagation direction is $\Gamma$ M. To perform modulation at $\lambda=1550 \mathrm{~nm}$ wavelength, $a$ is chosen to be $432 \mathrm{~nm}(1550 * 0.2788)$. In this condition, the displacement of the gap edge is expected to reach $7 \mathrm{~nm}(\sim$ 
$1,63 * 0.01 * 432)$ if a 0.01 variation of the refractive index is applied. The maximum extinction ratio that can be obtained is estimated to be $-22 \mathrm{~dB}\left(10 * \log _{10}\left(\mathrm{~T}_{\min }\right)\right)$.

The last part of this section is devoted to the predicted performances of the photonic structures when they are equipped with electrodes. The electrodes used for photonic crystal modulators are capacitive, as shown in figure 10 (a). So the bandwidth is limited by the length $L$ of the electrodes, which is fortunately very small. To give an order of value, $L=8 \mu \mathrm{m}$ in case where 21 rows of holes are arranged in a triangular array with $a=432 \mathrm{~nm}$ periodicity. The capacity of the electrodes is estimated to be $C=6 \cdot 2 \cdot 10^{-10} \mathrm{~F} / \mathrm{m}$ if the distance between electrodes is $d=10 \mu \mathrm{m}$, and if the holes are $1,5 \mu \mathrm{m}$ deep $(1.5 \mu \mathrm{m}$ is typically the depth that can be reached by FIB etching). This calculation was carried out by finite element method (femlab software). So the maximum bandwidth that can be expected with such a device is: $1 /(R * C * L)=4 \mathrm{THz}$, where $R=50 \Omega$. The photonic structure is thus compatible with high speed modulation.

The extinction ratio and the driving voltage are two other main important characteristics of the modulator. According to FDTD2D calculations, a $\Delta n_{e}=0,01$ amplitude of modulation of the extraordinary refractive index can yield a $-14 \mathrm{~dB}$ extinction ratio if the working wavelength is $\lambda=1550 \mathrm{~nm}$. Such a variation implies an effective electric field equal to $6.210^{7} \mathrm{~V} \cdot \mathrm{m}$, which is a hundred times higher than the effective electric field applied in standard modulators! This parameter has however to be considered in balance with other favourable factors. Firstly, the strong confinement of light in the photonic structure ensures a strong overlap coefficient $\eta$ between the electric and the optical field, which diminishes the required driving voltage (cf eq. (1)). Secondly, all the calculations have been carried out without taking into account the 3 dimensional aspects of the devices.

As a first conclusion, the theoretical results show the possibility of performing compact modulators based on photonic structures. The estimations of the performances are being pursued by $3 \mathrm{D}$ calculations to determine the driving voltage with more precision. But the first results justify the development of photonic structures in order to test their optical performances.

\subsection{Fabrication}

The chip was fabricated on a $0.3 \mathrm{~mm}$ thick single $\mathrm{X}$-cut $\mathrm{LiNbO}_{3}$ wafer. In a first step, an optical gradient index waveguide was fabricated by annealed proton exchange (APE). This step was realized through a $\mathrm{SiO}_{2}$ mask in benzoic acid at $180^{\circ} \mathrm{C}$ for 1.5 hours. The process was followed by an annealing of the optical waveguide at $333^{\circ} \mathrm{C}$ for 9 hours. These parameters were chosen in order to make the optical core as close as possible to the surface with a wavelength cut equal to $1.55 \mu \mathrm{m}$. With such experimental parameters, the optical core is estimated to be at $1.4 \mu \mathrm{m}$ from the surface, which can be compared to the $5 \mu \mathrm{m}$ depth that would result from a standard Ti-diffusion process.

The photonic crystals are then fabricated in the central region of the optical channel waveguide as shown in figure 10(a). The process of fabrication is detailed in ref. ${ }^{12}$ : the holes are etched in $\mathrm{LiNbO}_{3}$ substrate by focused ion beam (fib) milling. A 100nm thick $\mathrm{Cr}$ layer is previously deposited on the wafer to avoid charging effects. To fabricate the photonic structures, we used a FEI Dual Beam Strata 235, with $\mathrm{Ga}^{+}$gas and a 120pA probe current. The etching time of a $216 \mathrm{~nm}$-width hole is $3 \mathrm{~s}$ per hole. A SEM image of one of the fabricated structures is shown in figure 12 . The depth of the holes is estimated to be $1.5 \mu \mathrm{m}$ in these conditions.

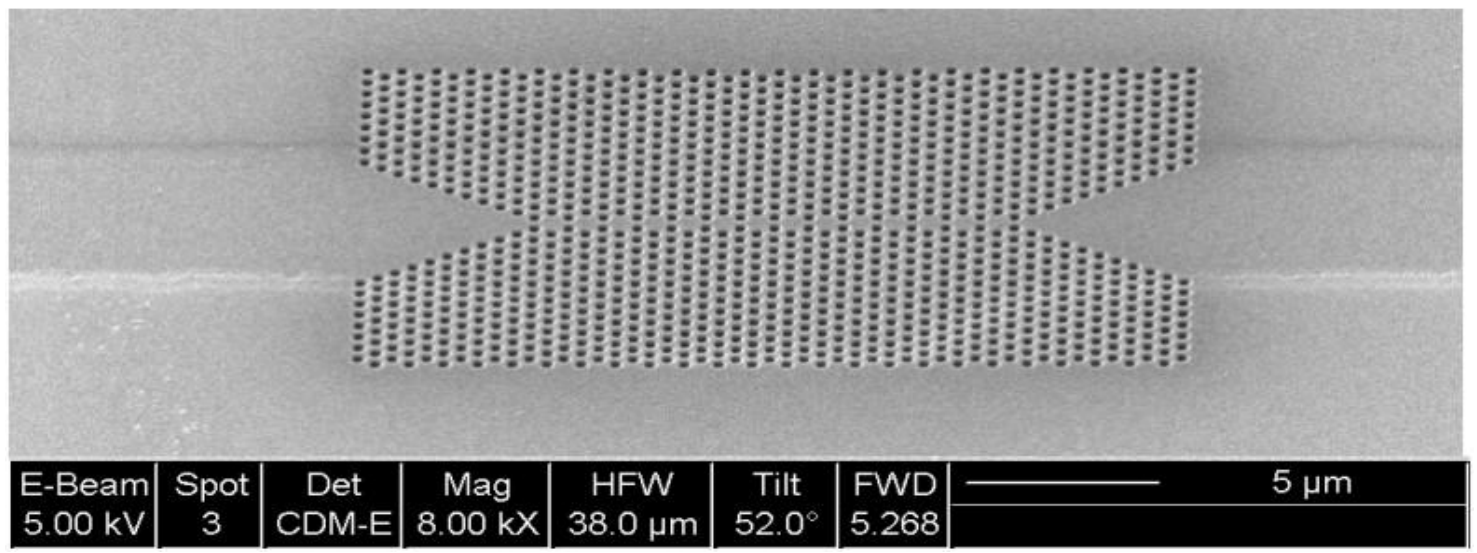

Fig. 12: $48 \times 26$ hexagonal lattice PCW1 and PCW3 structures to be characterised. 
Several kinds of structures were fabricated, with the objective of verifying the theoretical predictions. The purpose was also to investigate means of performing gaps with sharp edges. To our knowledge, we were the first to optically characterize a gap in a $\mathrm{LiNbO}_{3}$ photonic crystal ${ }^{13}$. These results were not very attracting for intensity modulation, because the edges of the characterized gap were very rough. The structures that are studied here are relative to the same array as described above (triangular arrangement of holes, ГМ direction of propagation), but there can be one or three lines of defects in the middle of the waveguide, which can theoretically enable peak transmissions. The period is adjusted in view of getting either a peak transmission or an edge of the gap at $1550 \mathrm{~nm}$ wavelength. In order to couple the annealed proton exchange (APE) mode ( $4 \mu \mathrm{m}$ size) to the photonic waveguides (approximately $500 \mathrm{~nm}$ and $1500 \mathrm{~nm}$ wide), the optical mode is smoothly guided through a photonic tapered structure (see Fig. 13). The details of the geometrical parameters are given in Table II.

\begin{tabular}{cccccc}
\hline \hline Structure & $\begin{array}{c}\text { Hole } \\
\text { depth }\end{array}$ & $\begin{array}{c}\text { Number of } \\
\text { rows }\end{array}$ & Hole diameter & Period & $\begin{array}{c}\text { Number of defect } \\
\text { Lines }\end{array}$ \\
\hline PCW0 & $1,5 \mu \mathrm{m}$ & 21 & $255 \mathrm{~nm}$ & $510 \mathrm{~nm}$ & 0 \\
\hline PCW1 & $1,5 \mu \mathrm{m}$ & 48 & $255 \mathrm{~nm}$ & $510 \mathrm{~nm}$ & 1 \\
\hline PCW3 & $1,5 \mu \mathrm{m}$ & 48 & $255 \mathrm{~nm}$ & $510 \mathrm{~nm}$ & 3 \\
\hline
\end{tabular}

Table II: Geometrical parameters of the triangular structures

The structures were first characterized by measuring their far field transmission. The experimental setup is shown in Fig. 13. The white light is generated by a sub-nanosecond microchip laser emitting at $1064 \mathrm{~nm}$ with $8 \mu \mathrm{J}$ energy per pulse $^{16}$. The laser light is launched into a photonic crystal (PC) fibre, which enhances the nonlinear effects required for the generation of a large super-continuum. The resulting output spectrum for a $20 \mathrm{~m}$ long PC fibre is shown in Fig. 13 (b).

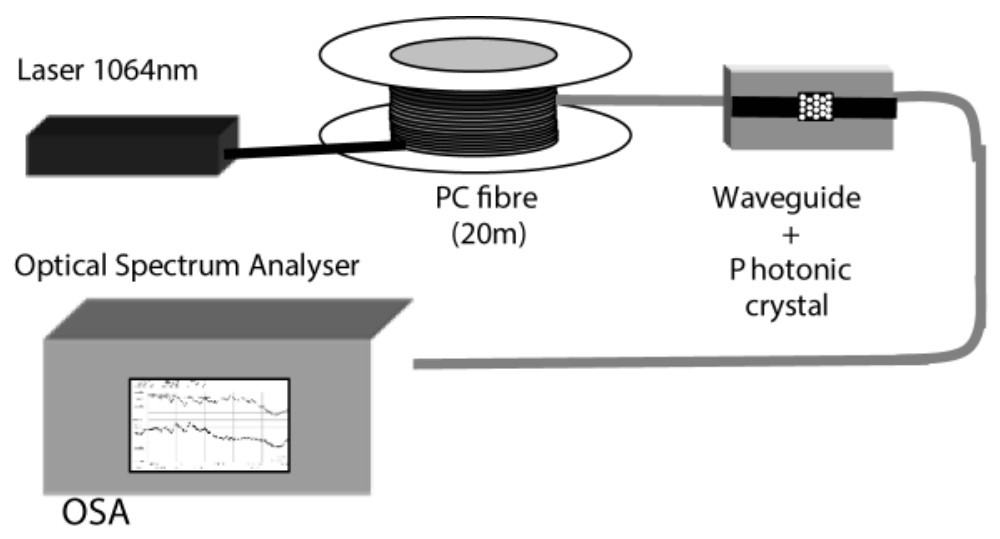

(a)

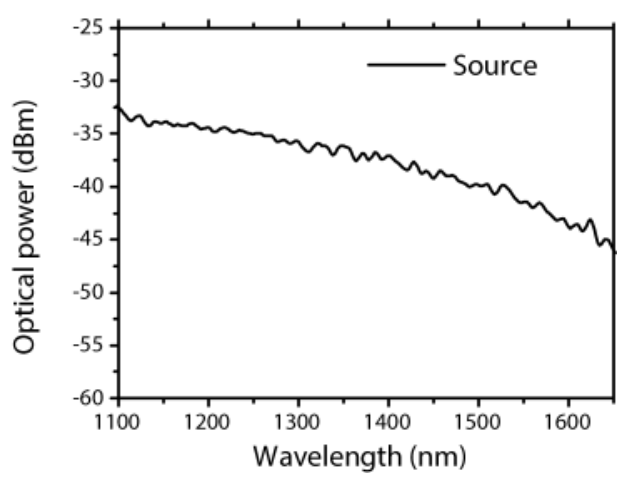

(b)

Figure 13: (a) experimental setup fo far field characterization, (b) Supercontinuum at the output of the PC fiber

The optical transmission was measured through the three photonic structures, and through a standard optical waveguide, fabricated on the same wafer and in the same conditions, as described above. The experimental results are shown in Fig. 14(a) and 14(b). As can be seen in the graph, the transmissions through the photonic structures (solid light black line in fig. 14(a), and (b), and circles in fig. 15(b)) exhibit a gap, which does not appear in the transmission through the single APE waveguide (dashed gray line in fig. 14(a) and filled black squares in fig. 14(b)). The extinction ratio is estimated to be lower than $-12 \mathrm{~dB}$. It cannot be evaluated more precisely because of the roughness of the transmission curves. The experimental gap lies from $1200 \mathrm{~nm}$ to $1600 \mathrm{~nm}$. So the position of the gap is experimentally confirmed, but there is no peak in PCW1 and PCW3 transmission responses. 


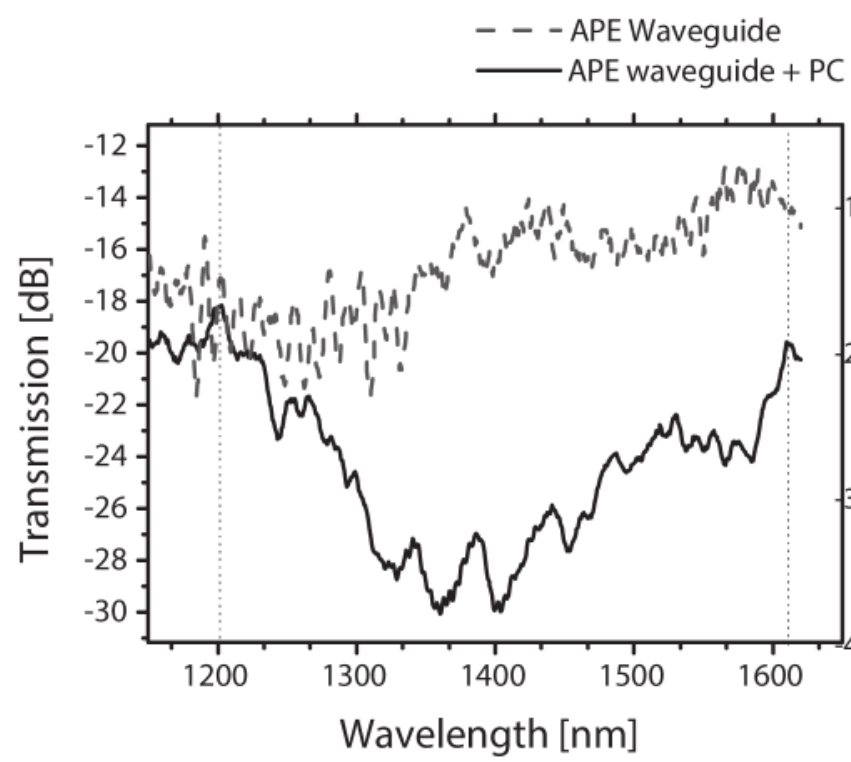

(a)

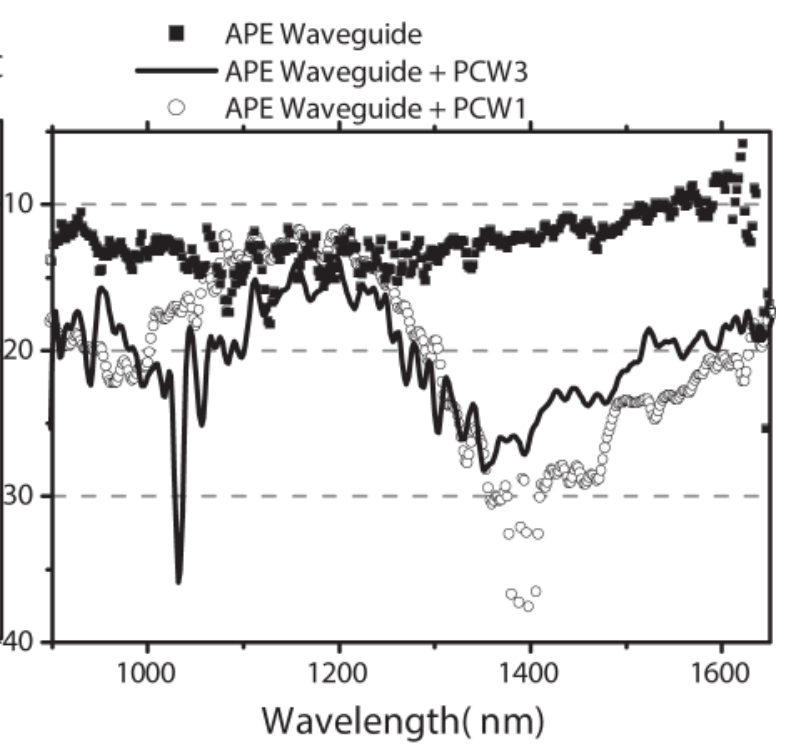

(b)

Figure 14: experimental transmission through photonic structures

(a) Transmission response through a PC structure integrated on an APE waveguide (solid black line)

(b) Transmission response through a PCW1 and a PCW3 structure, integrated on an APE waveguide (open circles and solid black line respectively)

For a further interpretation of the propagation of the light through the structures, we have then investigated the near field behaviour of the light inside the structures. In the work presented here, the instrument used is a commercial scanning near-field optical microscope (NT-MDT SMENA) in collection mode. To obtain the SNOM images, one needs to scatter the evanescent fields on the sample by raster scanning the subwavelength probe at a few nanometers from the surface. A non-optical shear force feedback is used to keep the probe at a constant distance from the sample surface as the sample is scanned beneath the probe. Both signals, the feedback and the optical one, are simultaneously acquired to construct topographic and SNOM collection images at the same sample locations.

Figure 15 shows the experimental set-up. To image the transmitted mode through the $\mathrm{LiNbO}_{3}$ photonic structure, two different laser sources and two different optical detectors have been utilized. The first acquisition has been performed at $810 \mathrm{~nm}$ (outside the PBG), with a Thorlabs STFC780 laser and detected with an Oriel 70680 photomultiplier. The second acquisition has been realized at $1.55 \mu \mathrm{m}$ with a distributed feedback laser OKI OL502OON and a InGaAs detector (Thorlabs D400FC), to characterize the propagating region inside the PBG.

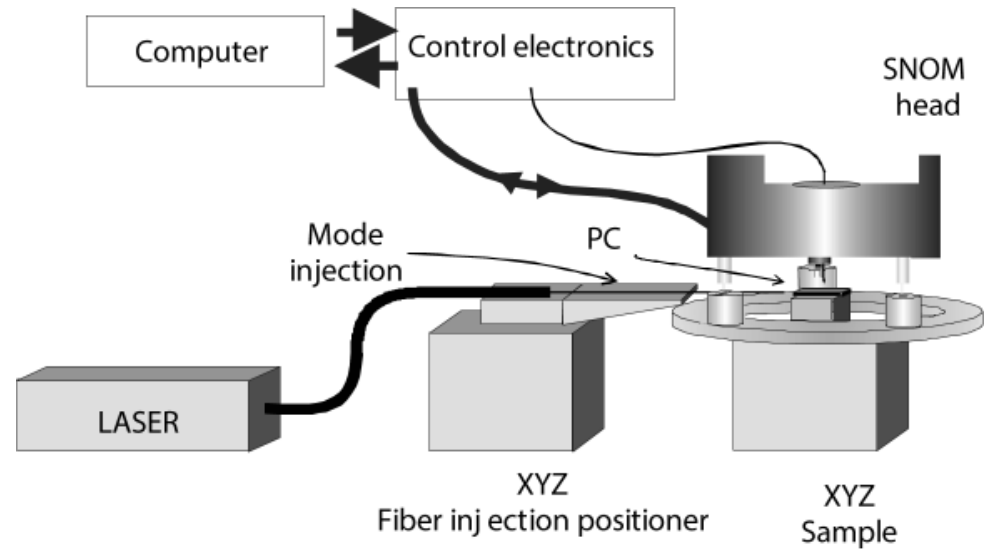

Figure 15: Schematics of the scanning near-field optical microscope working in collection mode. 
The optical image and topography of the PCW1 at 810nm is shown in Fig. 16. Fig. 16(a) shows the topography of the PCW1 structure and a zoom of a small region that consists of 7 holes. The depth of the hole measured by the SNOM tip is of the order of $30 \mathrm{~nm}$ which is far from the $1.5 \mu \mathrm{m}$ measured by the SEM image. This is basically due to the diameter of the hole, which is comparable in size as that of the tapered fibre. Fig. 16(b) shows the optical image of the light going through the PCW1 at $810 \mathrm{~nm}$. As measured by the transmission in Fig. 14, the optical transmission is of $-20 \mathrm{~dB}$ since the wavelength is outside the PBG of the photonic crystal.

We have also performed the near field measurements in a region inside the PBG in which an optical mode should propagate $(\lambda \sim 1.55 \mu \mathrm{m}$, transmission $\sim-25 \mathrm{~dB})$. Figure 17(a) shows the topography and $17(\mathrm{~b})$ the near field image respectively. It is worth mentioning that this set of measurements have been performed with a different SNOM probe than for the case of $810 \mathrm{~nm}$ due to tip destruction. Again, the topography shows clearly the photonic structure with a SNR of 10. We can very well appreciate the tapered beginning and end of the structure and the line of defects. The simultaneously recorded optical signal is shown in figure 17(b). This image corresponds to an input wavelength of $1.55 \mu \mathrm{m}$. The recorded signal shows clearly a confined mode that propagates through the line of defects. This propagating signal shows a distinct periodicity of $\lambda n_{\text {eff }}$ obtaining $n_{\text {eff }} 2.2, n_{\text {eff }}$ being the effective index of refraction corresponding to lithium niobate.

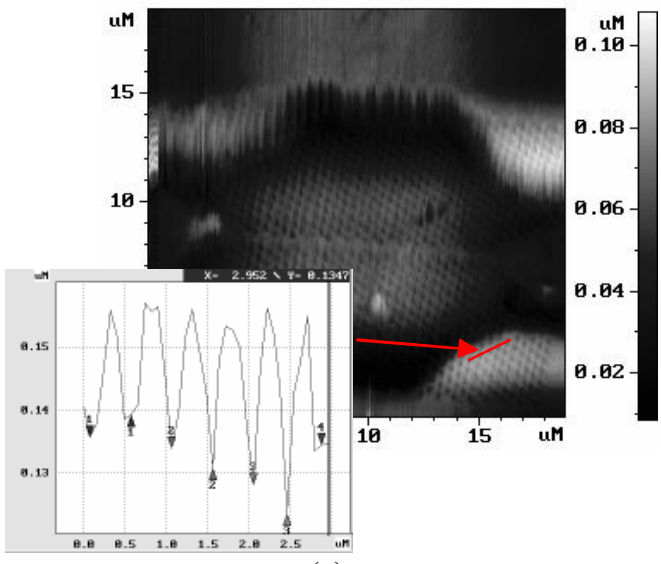

(a)

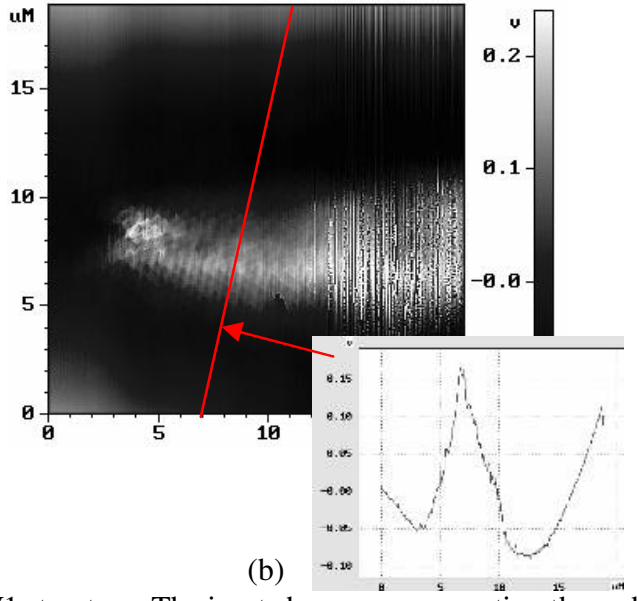

(b)

Figure 16: (a) Topographical image of 20x20 $\mu \mathrm{m}$ size of the PCW1 structure. The inset shows a cross section through 7 holes inside the structure. (b) The simultaneously recorded optical near field of the structure when the coupled wavelength is $810 \mathrm{~nm}$. The inset shows the width of the optical field inside the structure.

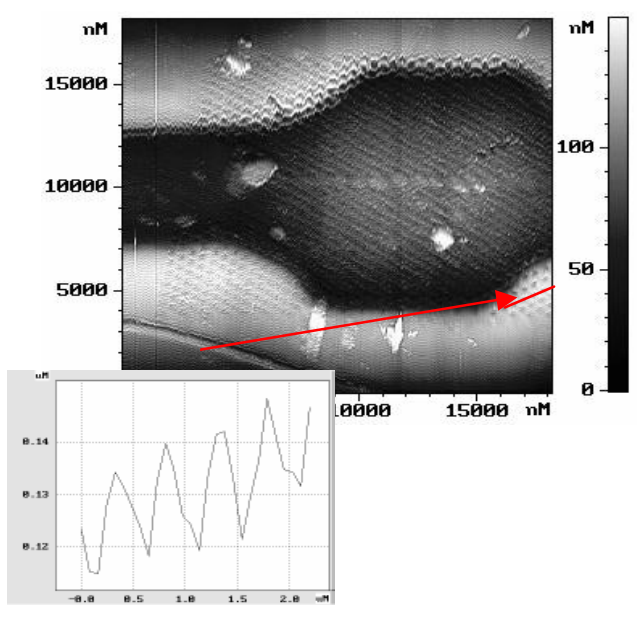

(a)
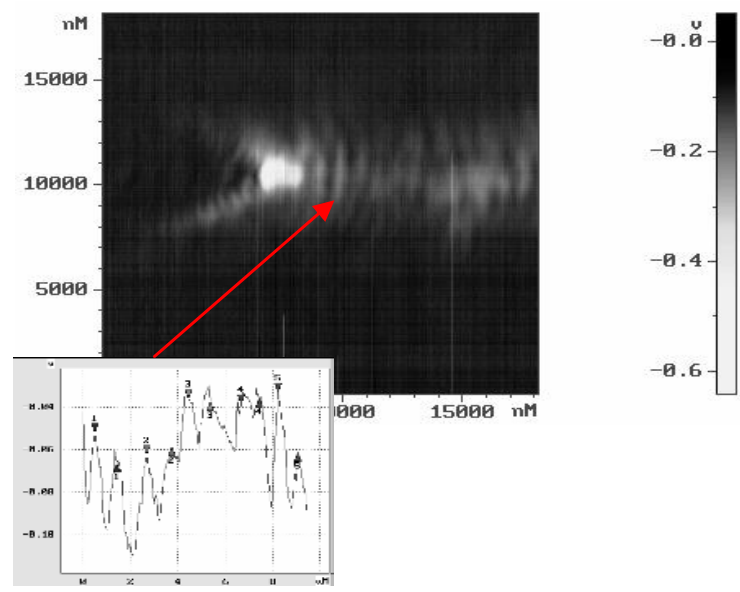

(b)

Figure 17: (a) Topographical image of 20x20 $\mu \mathrm{m}$ size of the PCW1 structure. The inset shows a cross section through 5 holes inside the structure. (b) The simultaneously recorded optical near field of the structure when the coupled wavelength is $1550 \mathrm{~nm}$. The inset shows the cross-section of the optical field along the propagation axes. 
We can then conclude that the near field characterization confirms the theoretical prediction according which the lines of defects should behave as photonic waveguides. However, the high attenuation through propagation explains why it cannot be visualized by far field characterization.

\section{CONCLUSION}

As a conclusion, we have reported two new trends on lithium niobate modulators. Using ferro-electric domain inversion combined with phase reversal electrodes, we have fabricated the first $10 \mathrm{~Gb} / \mathrm{s}$ single-drive $\mathrm{Z}$-cut $\mathrm{LiNbO}_{3}$ modulator with chirp as low as 0.07 and low driving voltage. A largely open eye diagram, $3.9 \mathrm{~dB}$ insertion loss, $25 \mathrm{~dB}$ static extinction ratio, $4.5 \mathrm{~V}$ static half-wave voltage and $14 \mathrm{GHz}$ bandwidth were simultaneously demonstrated. The low chirp, wide bandwidth and good extinction ratio of this device demonstrate its utility in long haul 10Gb/s applications. The frequency chirping was evaluated on a $1 \mathrm{GHz}-12 \mathrm{GHz}$ frequency range. The variation of the chirp parameter is 0.1 , which confirms the theoretical predictions. To avoid this harmful dispersion for rates higher than $40 \mathrm{~Gb} / \mathrm{s}$, we have presented solutions, based on several poled sections.

We have also developed an original solution to reduce the size of $\mathrm{LiNbO}_{3}$ modulators. The new configuration lies on a photonic structure that features an optical gap in the transmission response. We have shown both theoretically and experimentally the feasibility of photonic band gap structures in $\mathrm{LiNbO}_{3}$ materials. The possibility of modulating the output intensity has been evaluated, showing that a large bandwidth $(>30 \mathrm{GHz})$ can be expected. The experimental transmission response confirms the existence of an optical gap in the $\Gamma \mathrm{M}$ direction of propagation, with a $-12 \mathrm{~dB}$ extinction ratio. Scanning near field optical microscopy was performed at wavelengths inside and outside the PBG, reinforcing the predicted possibility of guiding the light through photonic structures. So this paper reports the first step toward micrometric $\mathrm{LiNbO}_{3}$ modulators. Electro-optical photonic structures are actually under test, and will be presented at the oral conference.

\section{ACKNOWLEDGMENTS}

The authors would like to acknowledge Cyril Billet, Nicolas Grossard and Jean-Yves Rauch for fruitful discussions, Blandine Guichardaz, Ludovic Gauthier-Manuel and denis Bitschène for technical assistance and particularly Eloïse Devaux from the Laboratoire desNanostructures ISIS, Université Louis Pasteur, Strasbourg, for the FIB fabrication.

\section{REFERENCES}

1. T. Kawanishi, K. Higuma, T. Fujita, J. Ichikawa, T. Sakamoto, S. Shinada, M. Izustu, J. Lightwave Technol., 23, 87-94, 2004

2. Y. C. Huang, K.W. Chang, Y.H. Chen, A.C. Chiang, T.C. Lin, B.C. Wong, J. Lightwave Technol., 20, 11651172,2002

3. J. Rodriguez-Asomoza, R. Rosas-Romero, S.E. Lobato-Larios, R. Alejos-Palomares, J.A. Navarro-Martinez, C. Gutierrez-Martinez, IEEE trans. instrum. meas., 54, 347-351, 2005.

4. S. Haxha, B.M.A. Rahman, R.J. Langley, Opt. Quantum. Electron., 36, 1205-1220, 2004

5. K. Aoki, J. Kondo, A. Kondo, T. Mori, Y. Mizuno, S. Shimodaira, M. Imaeda, Y. Kozuka, O. Mitomi, M. Minakata, IEEE Photon. Technol. Lett., 16, 2610-2612, 2004

6. J. Kondo, K. Aoki, A. Kondo, T. Ejri, Y. Iwata, A. Hamajima, T. Mori, Y. Mizuno, M. Imaeda, Y. Kozuka, O. Mitomi, M. Minakata, IEEE Photon. Technol. Lett., 17, 2077-2079, 2005

7. H. Kim, A.H. Gnauck, IEEE Photon. Tech. Letter, 14, 298-301, march 2002

8. G. Ghislotti, S. Balsamo, P. Bravetti, IEEE Photon. Technol. Lett. 15, 1534-1536, 2003

9. S. Oikawa, F. Yamamoto, J. Ichikawa, S. Kurimura, K. Kitamura, J. Lightwave Technol., 23, 2756-2760, 2005

10. N. Courjal, H. Porte, A. Martinez, J.P. Goedgebuer, IEEE Photon. Technol. Lett., 1509-1512, 2002

11. N. Courjal, H. Porte, J. Hauden, P. Mollier, N. Grossard, J. Lightwave Technol., 22, 1338-1343, 2004

12. W.V. Sorin, K.C. Chang, G.A. Conrad, and P.R. Hernday, J. Lightwave Technol., 10, 787-793, 1992

13. S. Yin, Microwave and optical technology letters 22, 396-399, 1999.

14. F. Lacour, N. Courjal, M.-P. Bernal, A. Sabac, C. Bainier, M. Spajer, Opt. Mat. 27, 1421-1425 2005.

15. M. Roussey, M.-P. Bernal, N. Courjal, F.I. Baida, Appl. Phys. Lett, 87, 241101, 2005

16 K.P. Hansen, R.E. Kristiansen, Supercontinuum generation in photonic crystal fibers, Application Note, Crystal Fibre

*nadege.bodin@univ-fcomte.fr 